\title{
Experimentation with an adaptive waterfall controller with disturbance effect
}

\author{
Nino Vega ${ }^{1}$, Ms.C, Pablo Parra, $\mathrm{PhD}^{2}$, Luis Córdova ${ }^{1}$, Ms.C, Joselyne Andramuño ${ }^{1}$ \\ ${ }^{1}$ Universidad Católica de Santiago de Guayaquil,nino.vega@cu.ucsg.edu.ec, luis.cordova@cu.ucsg.edu.ec, \\ joselyne.andramuno@cu.ucsg.edu.ec \\ ${ }^{2}$ Universidad Católica de Santiago de Guayaquil, Ecuador, pablitoparrarosero@gmail.com
}

\begin{abstract}
The control systems whose output variable is slow requires more efficient control actions, in this line, there are the cascade-type controllers, which prevent the disturbances in the input of the process from manifesting in the output, currently the analysis are reflected in many investigations, but in a simulated way, and there are a few studies where the implementation is carried out at the didactic level, which allows to perform the verification of the simulations, and to observe the effect that this type of control has when is going to work in a real way, so we can prove the theories and affirmations of the scientific community. In this research, we complete the development of an adaptive cascadetype controller in the two control loops, in a didactic equipment that allows perturbations. To achieve the objectives, we obtained the mathematical modeling of the system, the controllers were designed, the control algorithm is implemented in a plant, whose variables are level and flow, and the effect that these controllers have on perturbations that are made to the process variables. During the implementation of the control algorithm, it was possible to verify that these types of controllers cause that the perturbations that are made at the input of the process were minimun reflected in the output variable.

Keywords- Adaptive Control, Cascade, Didactic, Disturbances.
\end{abstract}

Digital Object Identifier (DOI):

http://dx.doi.org/10.18687/LACCEI2019.1.1.266

ISBN: 978-0-9993443-6-1 ISSN: 2414-6390

$17^{\text {th }}$ LACCEI International Multi-Conference for Engineering, Education, and Technology: "Industry, Innovation, And Infrastructure for Sustainable Cities and Communities”, 24-26 July 2019, Jamaica. 


\title{
Experimentation with an adaptive waterfall controller with disturbance effect
}

\author{
Nino Vega ${ }^{1}$, Ms.C, Pablo Parra, $\mathrm{PhD}^{2}$, Luis Córdova1, Ms.C, Joselyne Andramuño ${ }^{1}$ \\ 1Universidad Católica de Santiago de Guayaquil,nino.vega@cu.ucsg.edu.ec, luis.cordova@cu.ucsg.edu.ec, \\ joselyne.andramuno@cu.ucsg.edu.ec \\ ${ }^{2}$ Universidad Católica de Santiago de Guayaquil, Ecuador, pablitoparrarosero@gmail.com
}

\begin{abstract}
The control systems whose output variable is slow requires more efficient control actions, in this line, there are the cascade-type controllers, which prevent the disturbances in the input of the process from manifesting in the output, currently the analysis are reflected in many investigations, but in a simulated way, and there are a few studies where the implementation is carried out at the didactic level, which allows to perform the verification of the simulations, and to observe the effect that this type of control has when is going to work in a real way, so we can prove the theories and affirmations of the scientific community. In this research, we complete the development of an adaptive cascade-type controller in the two control loops, in a didactic equipment that allows perturbations. To achieve the objectives, we obtained the mathematical modeling of the system, the controllers were designed, the control algorithm is implemented in a plant, whose variables are level and flow, and the effect that these controllers have on perturbations that are made to the process variables. During the implementation of the control algorithm, it was possible to verify that these types of controllers cause that the perturbations that are made at the input of the process were minimun reflected in the output variable.
\end{abstract}

Keywords-Adaptive Control, Cascade, Didactic, Disturbances.

\section{INTRODUCCIÓN}

En la mayoría de las investigaciones que se han realizado, para obtener la ecuación matemática que represente el sistema, se aísla el proceso de las perturbaciones e incertidumbres en los parámetros de los componentes involucrados en el control, por tal razón, la función matemática del proceso a controlar es una aproximación de la realidad. Al tener un modelo aproximado del proceso, el diseño del algoritmo de control no es el correcto, lo que trae como consecuencia que los profesionales del área tengan que realizar reajustes de los parámetros de control de manera práctica. Para solucionar estos inconvenientes en[1], se desarrolló un controlador en cascada, controladores que también son utilizado para mejorar las perturbaciones que se presenten[1] [2] en la variable de salida, este esquema de control utiliza un sensores intermedio y por lo menos dos lazos de control, en donde el lazo de control interno, toma como punto de operación la señal de control del lazo externo, como lo realizan [3] [4] con lo que mejoran el control de un sistema. Una variación del controlador cascada es el adaptativo cascada el cual ha demostrado presentar mejores prestaciones que los controladores modernos, como los controladores difuso de variable intermedia FCIV y el control de temperatura de un sistema de calefacción solar [5][6]. y también los desarrollo híbridos como el presentador por [7] en donde el lazo de control interno es multivariable y el externo es predictivo, y en [8] un

Digital Object Identifier (DOI):

http://dx.doi.org/10.18687/LACCEI2019.1.1.266

ISBN: 978-0-9993443-6-1 ISSN: 2414-6390 control cascada IMC se desarrolla para controlar la temperatura del vapor en un caldera en la que los resultados obtenidos del controlador adaptativo cascada son efectivos en el proceso.

Actualmente debido a la robustez del control adaptativo cascada se los está utilizando en diferentes áreas tales como: reguladores de tensión[9],convertidores CC-CC, en inversores fotovoltaicos como los de [10], en procesos químicos como los de [11] en donde usan un control para regular la concentración de salida frente a perturbaciones en la alimentación y variación de entalpia de reacción en un reactor tubular, en este proceso se utiliza como variable de control interna, la concentración, y dos lazos de control externo, para mantener la temperatura en niveles deseados, los resultados encontrados en esta investigación fueron satisfactorios.

En la parte educativa se ha desarrollado controladores en cascada como[12] en la universidad politécnica de Chimborazo en donde se desarrolla y experimenta con un viscosímetro saybolt se hace control cascada de temperatura. En el Centro de Electricidad y Automatización Industrial en Cali Colombia CEAI - SENA, se diseñó e implementó de un sistema de control de la Planta Didáctica de Intercambio Térmico - PIT000, El sistema de control es basado en una estrategia cascada temperatura-flujo, implementada mediante el uso de tecnología de instrumentación y control industrial[11].

En la línea de robótica se usa este tipo de control para el seguimiento y estabilización del sistema robótico para mejorar las perturbaciones que se presentan [13]. De todas estas aplicaciones didácticas son escasas las presentación de investigaciones, en donde se cuente con una planta, donde se pueda realizar pruebas a los controladores adaptativo en cascada funcionando en situaciones adversas en donde a la variable de salida, se le realice perturbaciones, tanto a la entrada como a la salida del proceso, y se pueda apreciar la robustez de estos controladores.

En lo que respeta a las aplicaciones de vehículos autónomos se tiene desarrollos de control cascada como los de [14], en la que se realiza un controlador predictivo MPC para la dinámica vehicular y longitudinal de un vehículo autónomo las simulaciones realizadas demuestran un mejor rendimientos de este tipo de control, en [15] se lo utiliza para dirigir un vehículo en control cinemático , en esta aplicación se lo realiza un control cascada, el algoritmo desarrollado mejora el seguimiento de la recta establecida.

Al nivel industrial se ha utilizado en aplicaciones como: en plantas de intercambio término[16], en el que utilizan un sistema embebido para la implementación del controlador el algoritmo de control es colocado en el sistema embebido, para controlar motores generadores[17],en reactores tubulares[18]. 
En telecomunicaciones también se han desarrollado controladores adaptativo cascada como los de [19], en el que se desarrolla un filtro hibrido adaptativo para el arreglo lineal uniforme de antenas para generar lóbulos de radiación

Adicional a todas las aplicaciones del control adaptativo cascada se han desarrollado algunas aplicaciones aisladas como el control adaptativo cascada por modelo de referencia [20],otro desarrollo de control cascada difuso de nivel y flujo, en una planta real, en la que se verifica que mejora el rendimiento de la señal de control, y la estabilidad de la variable principal de control [21], en automovilístico se tiene la de [22] en el freno por cable, el sistema se compone por un actuador electrónico un cilindro maestro y un freno hidráulico de manera experimental se valida la propuesta.

Con todos los trabajos desarrollados del control en cascada y del adaptativo cascada, surge la necesidad a nivel didáctico de verificar todos los efectos de este control, para lo cual, se requiere de una planta, en la que se puedan realizar diversos tipos de perturbaciones, tanto a la entrada como a la salida de la planta, en donde el usuario tenga la opción de comprobar algunas de las afirmaciones dadas por los diferentes autores, tales como: mejora en la acción de control, y en presencia de perturbaciones, así como también bajar los costos del implementación, en base a los sistemas embebidos, teniendo como precedente que la mayoría de las investigaciones realizadas, han desarrollado este tipo de control de manera simulada, y con tecnología como los PLC, Tarjetas FPGA lo que hace que los costos de implementación aumenten .

La estrategia de control adaptativo que se implementara, es tipo de ganancias programadas, en donde en el lazo de control interno que se controle es caudal y en el lazo externo nivel de un tanque.

En el desarrollo de los objetivos de esta investigación, se utilizará la maqueta dada por[23], en donde se tomarán datos experimentales de la planta, que permitan obtener la ecuación lineal y no lineal tanto del tanque de control y del actuador, relaciones caudales vs nivel y voltaje vs caudal, respectivamente. Con el uso del simulador se calibrará los controladores a utilizar en los diferentes puntos de operación, para los dos lazos de control adaptativo cascada. Finalmente se desarrollara el algoritmo de control, el mismo que será colocado en el sistema embebido para que este realice los cálculos de la variable de control para enviarle a la planta real.

\section{MATERIALES Y MÉTODOS}

Para realizar las pruebas del control adaptativo cascada, se utilizara la planta didáctica construida en [23], la misma que está compuesta por un tanque de almacenamiento, otro tanque donde se realizara el control de nivel, una bomba para ingresar agua al sistema, dos válvulas en la entrada y dos válvulas en la salida del tanque de control, de las cuales, una es para funcionamiento normal y la otra es para realizar las perturbaciones de las variables del lazo externo e interno del sistema. Además, para realizar la retroalimentación de los lazos de control se tienen dos sensores un de caudal y otro para medir el nivel, el diagramas de esta maqueta se muestran en la figura 1.

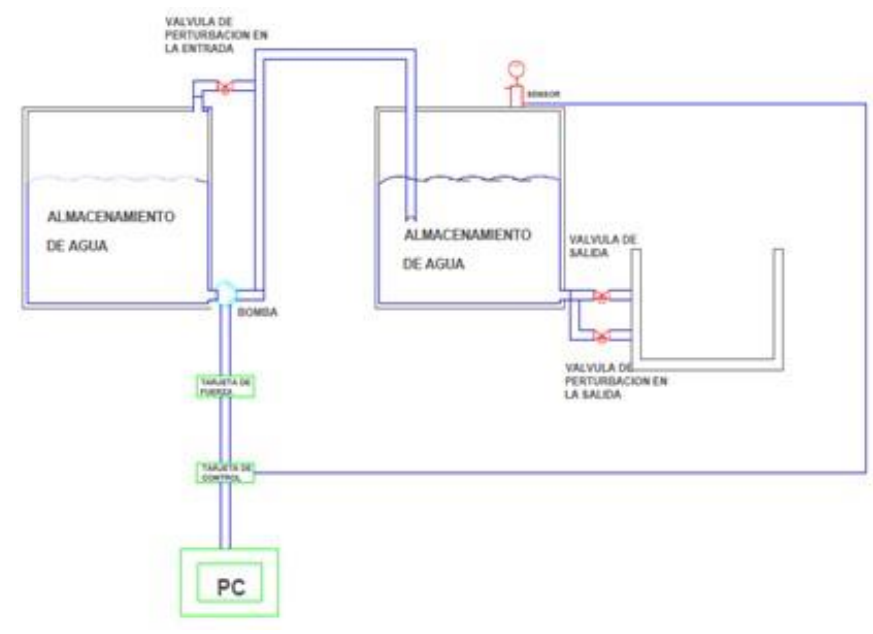

Fig. 1 Diagrama planta control cascada.

\section{A. Hardware del Sistema}

El hardware a utilizar en este prototipo se basa en el desarrollo dado en [24]. En donde el control del equipo de fuerza, se lo desarrolla con una tarjeta de fuerza PWM, y el algoritmo de control se lo embebe en una tarjeta Ardunino Mega 256. En la figura 2 Se muestra el diagrama de conexiones del circuito eléctrico, en la que se notan los dos sensores: el de nivel, (utilizado por el controlador externo), y el sensor de caudal, (utilizado para el control interno).

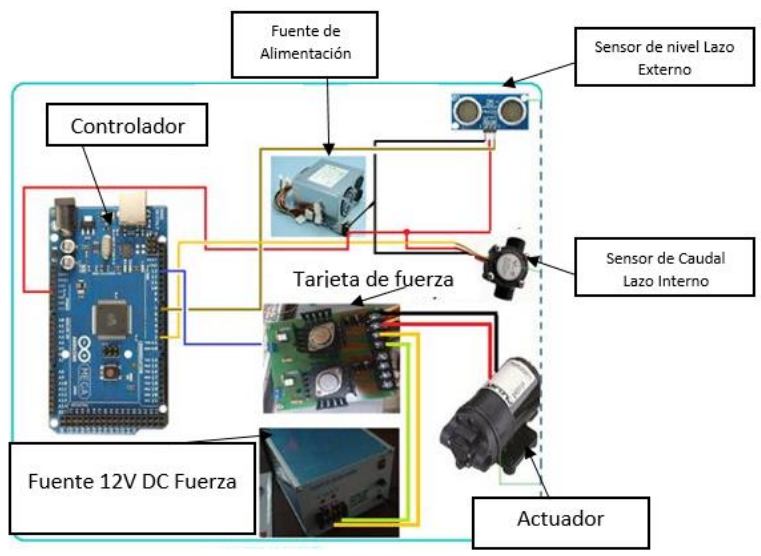

Fig. 2 Circuito Eléctrico planta control adaptativo cascada con variables nivel y caudal

\section{MODELAMIENTO MATEMÁTICO}

A. Modelamiento del tanque de control

$17^{\text {th }}$ LACCEI International Multi-Conference for Engineering, Education, and Technology: "Industry, Innovation, And Infrastructure for Sustainable Cities and Communities", 24-26 July 2019, Jamaica. 
El modelamiento matemático del sistema se lo realiza en dos partes: uno para obtener la ecuación del actuador ,y otro para obtener la ecuación del tanque de control, si se analiza las ecuaciones del tanque de la figura 1, este tiene una entrada continua de caudal qi, y como salida un caudal qo, la ecuación diferencia que relaciona la entrada con la salida del tanque es la ecuación 1, se las tomó de [25] [26][27], las cuales se basan en la relación de balance de masa entre el caudal que entra, el que sale, y lo que se acumula dentro del tanque .

$$
q i-q o=A \frac{d h}{d t}
$$

El valor de A corresponde a la sección trasversal del tanque de control $(510 \mathrm{~cm} 2), \frac{d h}{d t}$ variación de nivel dentro del tanque, $\boldsymbol{q} \boldsymbol{i}$ es el caudal de ingreso, qo el caudal de salida, el mismo que puede ser de tipo laminar o turbulento, su ecuación es $\boldsymbol{q o}=\boldsymbol{K}_{\boldsymbol{v}} * \boldsymbol{h}$ para flujo laminar, o $\boldsymbol{q o}=\boldsymbol{K}_{\boldsymbol{v}} * \sqrt{\boldsymbol{h}}$, para flujo turbulento. Para seleccionar la ecuación de qo, se tomaron datos experimentales y se determinó el número de Reynolds que según [28] es el que determina si el flujo el laminar o turbulento (ecuación 2), los valores obtenidos corresponden a 16302 y 6751, para la máxima y mínima capacidad de la bomba respectivamente, con este valor la ecuación a utilizar para qo es de tipo turbulento.

Al reemplazar qo en la ecuación 1 se genera la ecuación 3, la misma que es de tipo no lineal, el valor de $K_{v}$ es una constante de la válvula, al linealizar con Taylor alrededor de un en un punto de operación se obtiene la ecuación 4 .

$$
R e=\frac{\rho * D * v}{\eta}
$$

Con los datos de la planta:

$\rho=$ es la densidad del agua

$\mathrm{D}=$ diámetro de la tubería $(16 \mathrm{~mm})$

$v=910,44 \mathrm{~cm} / \mathrm{s}$ o $412.935 \mathrm{~cm} / \mathrm{s}$ es la velocidad mínima y

máxima sobre las tuberías $\eta=0.000891 \mathrm{~kg} / \mathrm{m}_{s}$ Viscosidad del fluido (agua a 25 grados centígrados)

$A \frac{d h}{d t}=Q i-K_{v} * \sqrt{h}$

$\mathrm{A} \frac{d\left(h-h_{o}\right)}{d t}=\left(q i-q i_{o}\right)-K_{v} * \frac{1}{2 * \sqrt{h_{o p}}}\left(h-h_{o}\right)$

Donde $h_{o}$ es el valor de altura deseado, al realizar los cambios de variable en la ecuación 4 por:

$Q=q i-q i_{o}$

$H=h-h_{o}$

$\mathrm{K}=K_{v} * \frac{1}{2 * \sqrt{h}}$

Se obtienen la ecuación 5 .
$\mathrm{A} \frac{d H}{d t}=Q-K H$

Al aplicar la transformada de Laplace se genera las ecuaciones 6 y 7 :

$A S H(s)+K H(s)=Q(s)$

$G(s)=\frac{H(s)}{Q(s)}=\frac{1}{A s+K}$

Al normalizar la ecuación 7 se obtiene la ecuación 8:

$$
G(s)=\frac{\frac{1}{A}}{s+\frac{K}{A}}
$$

Las constantes de la ecuación 8 se las obtiene realizando pruebas experimentales en lazo abierto siguiendo los lineamientos dados en [29], se ingresar agua al tanque de control y esperando que el nivel se estabilice, en estas condiciones, se almacenaron los datos como: altura $\mathrm{h}$, el caudal de la bomba, y el código PWM enviado a la tarjeta de fuerza. Con los datos almacenados se calcula el valor de $K_{v}$, utilizando la ecuación 3, con $\frac{d h}{d t}=0$, y finamente se reemplazan los valores en la ecuación 8 , dando la función de transferencia de varios puntos de operación como en [30].

\section{B. Modelamiento no Lineal del tanque de control}

La ecuación no lineal de la planta se la representa con dos ecuaciones (ecuación 8 y 9), para obtener la ecuación 8 , se utiliza los 70 datos experimentales de la prueba anterior, tabla 1, y en Matlab por el método de mínimos cuadrados se obtienen un polinomio de grado 4 que aproxime los datos altura vs caudal.

$h=a * q_{i}-b * q_{i}{ }^{2}+c * q_{i}{ }^{3}+d * q_{i}{ }^{4}+e$

Los valores de las constantes de la ecuación 8 corresponden:

$a=8.33998 b=1.26372 \quad c=0.00859 d=0.00002$

$e=2083.10268$

Para considerar el estado transitorio del sistema nivel vs caudal del tanque de control, se lo considera como un sistema de grado uno, (ecuación 9), en donde la entrada del sistema es el nivel h (ecuación 8), y la salida será el nivel h2. Para determinar las constantes $\mathrm{k} 1 \mathrm{y} \mathrm{k} 2$, se realiza pruebas experimentales en lazo abierto al tanque de control, se le ingresa $111.11 \mathrm{~cm} 3 / \mathrm{s}$ de caudal, y se determina el tiempo que demora el sistema en llegar a estado estable (aproximadamente 36 minutos), con este valor se utilizó el simulador simulink de Matlab para obtener estas constantes, donde k1 y k2 es de 0.0029 .

$17^{\text {th }}$ LACCEI International Multi-Conference for Engineering, Education, and Technology: "Industry, Innovation, And Infrastructure for Sustainable Cities and Communities", 24-26 July 2019, Jamaica. 


$$
\frac{d h 2}{d t}+k 1 * h 2=k 2 * h
$$

\section{Modelamiento no Lineal del Actuador}

La ecuación no lineal del actuador corresponde a las ecuaciones 10 y 11. Para obtener la ecuación 10, se procede de la misma manera que de la ecuación del tanque de control, pero para este caso, se tomaron datos experimentales de la planta como en [31], en donde se coloca un valor de voltaje a la bomba y se obtiene el valor del caudal estabilizado con estos datos se obtiene la ecuación en estado estable de la relación voltaje de la bomba (V) vs el caudal de salida (Q), con estos datos se obtuvo la ecuación 10 .

$$
\begin{aligned}
Q=11.4960 * \mathrm{v}+ & 1.1816 v^{2}-0.1236 * v^{3}+0.0041 * v^{4} \\
& -0.6203
\end{aligned}
$$

Si se considera el transitorio del actuador se puede seguir la metodología dada por [32][33], pero en este caso se lo considerará como un sistema de grado uno, ecuación 11.

La ecuación toma como entrada el caudal (Q) ecuación 10, y como salida el caudal Q2. Para obtener las constantes k3 y k4, se tomó datos experimentales en lazo abierto, colocando un voltaje de 12 voltios a la bomba y midiendo el tiempo que demora en estabilizarse el caudal, los valores obtenidos son $183 \mathrm{~cm} 3 / \mathrm{s}$ y 2 segundos respectivamente, con estos datos, se utilizó el simulador simulink de Matlab en las que se colocó las ecuaciones 9 y 10 y se obtuvo que las constates $\mathrm{k} 3=\mathrm{k} 4=5.2$

$\frac{d Q 2}{d t}+k 3 * Q 2=k 4 * Q$

\section{Diseño DEL CONTROL ADAPTATIVO EN CASCADA}

\section{A. Diseño del Controlador Adaptativo De lazo Interno}

La estrategia de control adaptativo que se utiliza en esta investigación, está dada por [34], que consiste en obtener los controladores PID para diferentes puntos de operación de cada modelo, y conforme la variable de control cambie, el algoritmo selecciona las ganancias a utilizar en el control dependiendo del punto de trabajo.

El modelo del actuador, ecuaciones 9 y 10, se colocan en Simulink, y con este se obtienen las constantes del controlador PI de cada punto de operación, y se verifica el rango de operación que puede cubrir en cada caso en la Tabla I. Se consolida la información de las constantes a utilizar en el control.

\section{B. Diseño del Controlador Adaptativo De lazo Externo}

De la misma manera como se obtuvo la tabla uno, se procede a calibrar el controlador PID para el lazo externo, pero colocando en funcionamiento el lazo interno con los datos de la tabla..., las constantes para cada rango de operación de los controladores PID externos se muestran en la Tabla II y en la figura 3 se presenta el diagrama del control cascada usado en la calibración.
TABLA I

CONTROLADORES PI PARA DIFERENTES PUNTOS DE OPERACIÓN DE CAUDAL

\begin{tabular}{|c|c|c|c|}
\hline \multicolumn{5}{|c|}{ CONTROLADOR PI DE LA VARIABLE CAUDAL } \\
\hline $\begin{array}{c}\text { Zonas de } \\
\text { Operación }\end{array}$ & $\begin{array}{c}\text { Rango de } \\
\text { Funcionamiento }\end{array}$ & $\begin{array}{c}\text { Constant } \\
\text { e Kp }\end{array}$ & $\begin{array}{c}\text { Constant } \\
\text { e Ki }\end{array}$ \\
\hline Zona 1 & $\begin{array}{c}0 \mathrm{~cm}^{3} / \mathrm{s}<=\text { Caudal }<30 \\
\mathrm{~cm}^{3} / \mathrm{s}\end{array}$ & 0.50 & 0.65 \\
\hline Zona 2 & $\begin{array}{c}30<=C \text { Caudal }<60 \\
\mathrm{~cm}^{3} / \mathrm{s}\end{array}$ & 0.67 & 3 \\
\hline Zona 3 & $\begin{array}{c}60<=\mathrm{Caudal}^{3}<90 \\
\mathrm{~cm}^{3} / \mathrm{s}\end{array}$ & 0.2 & 1 \\
\hline Zona 4 & $\begin{array}{c}90<=C \mathrm{Caudal}^{2}<120 \\
\mathrm{~cm}^{3} / \mathrm{s}\end{array}$ & 0.75 & 0.73 \\
\hline Zona 5 & $\begin{array}{c}120<=\mathrm{Caudal}<160 \\
\mathrm{~cm}^{3} / \mathrm{s}\end{array}$ & 0.4 & 0.49 \\
\hline
\end{tabular}

TABLA II

CONSTATES DEL CONTROLADOR PID NIVEL

\begin{tabular}{|l|l|l|l|l|}
\hline CONTROLADOR PID DE LA VARABLE NIVEL \\
\hline $\begin{array}{l}\text { Zonas } \\
\text { de } \\
\text { Operaci } \\
\text { ón }\end{array}$ & $\begin{array}{l}\text { Rango fe } \\
\text { Funcionamie } \\
\text { nto }\end{array}$ & $\begin{array}{l}\text { Consta } \\
\text { nte Kp }\end{array}$ & $\begin{array}{l}\text { Consta } \\
\text { nte Ki }\end{array}$ & $\begin{array}{l}\text { Consta } \\
\text { nte Kd }\end{array}$ \\
\hline Zona 1 & $\begin{array}{l}30<\text { Nivel }<39 \\
\mathrm{~cm}\end{array}$ & 30 & 0.18 & 20 \\
\hline Zona 2 & $\begin{array}{l}30<=\quad \text { Nivel } \\
<25 \mathrm{~cm}\end{array}$ & 28.5 & 0.25 & 18 \\
\hline Zona 3 & $\begin{array}{l}25<=\quad \text { Nivel } \\
<20 \mathrm{~cm}\end{array}$ & 25.2 & 0.368 & 24 \\
\hline Zona 4 & $\begin{array}{l}20<=\quad \text { Nivel } \\
<15 \mathrm{~cm}\end{array}$ & 35 & 0.5 & 40 \\
\hline Zona 5 & $\begin{array}{l}15<=\quad \text { Nivel } \\
<10 \mathrm{~cm}\end{array}$ & 120 & 0.9 & 37 \\
\hline Zona 6 & $\begin{array}{l}10<=\quad \text { Nivel } \\
<5 \mathrm{~cm}\end{array}$ & 115 & 7 & 40 \\
\hline
\end{tabular}

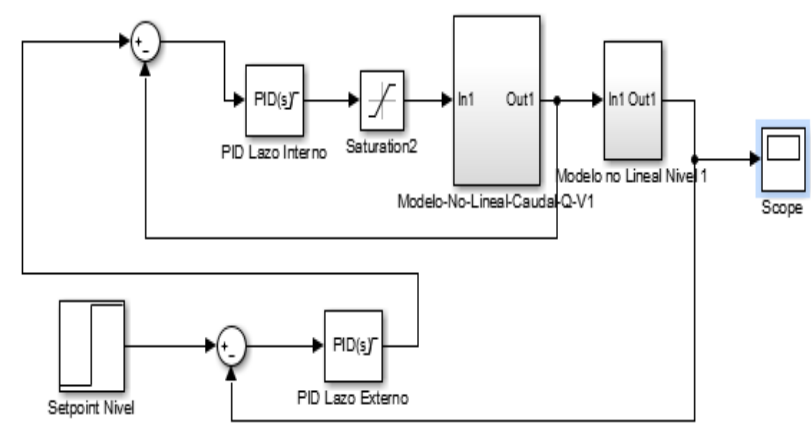

Fig. 3 Diagrama de bloque del control cascada.

\section{Diseño Del Algoritmo de Control en CASCADA}

Se desarrolló un programa que cumple las siguientes acciones: primeramente, se realiza una declaración de todas las 
variables, se configura los pines de la tarjeta de control. Luego de esto se ingresa a un lazo de control, en donde se calcula el tiempo de muestreo para el lazo de control interno (1 segundo), si se ha cumple el tiempo de muestreo, entonces ejecuta la acción de control cascada, lazo interno, caso contrario espera hasta cumplir este tiempo. Dentro de la acción de control adaptativo del lazo interno, primero se toma lectura del sensor de caudal, con este valor se selecciona de la tabla I, las constantes del controlador PI que se debe aplicar en la ley de control, cuya salida se la pasa por un saturador, en función de los límites del actuador (valor de caudal de 0 y $183 \mathrm{~cm} 3 / \mathrm{s}$ en este caso), este valor modificado, será el que se aplica al actuador en valores correspondientes en voltios.

Terminada la aplicación del controlador interno, el algoritmo realiza una supervisión si se ha cumplido el tiempo de muestreo del lazo externo (4.5 segundos), si no se ha cumplido, el programa regresa para aplicar nuevamente la ley de control del lazo interno. Si se ha cumplido el tiempo de muestreo del lazo externo, el programa entra a la aplicación del control adaptativo de este lazo externo, para el cual, primero lee el sensor de nivel, con este valor selecciona de la tabla II las constante del controlador PID que debe aplicar, calcula la respuesta de control (u), y este valor se lo envía como setpoint del lazo interno.

\section{RESUltados Y Discusión}

Al sistema mostrado en la figura 1 se le aplica el algoritmo de control adaptativo, pero sin habilitar el lazo de control interno, en la prueba realizada se hace pasar la planta por diferentes puntos de operación $(0 \mathrm{~cm}-8 \mathrm{~cm}-25 \mathrm{~cm}-17 \mathrm{~cm})$, los resultados se muestran en la figura 6.

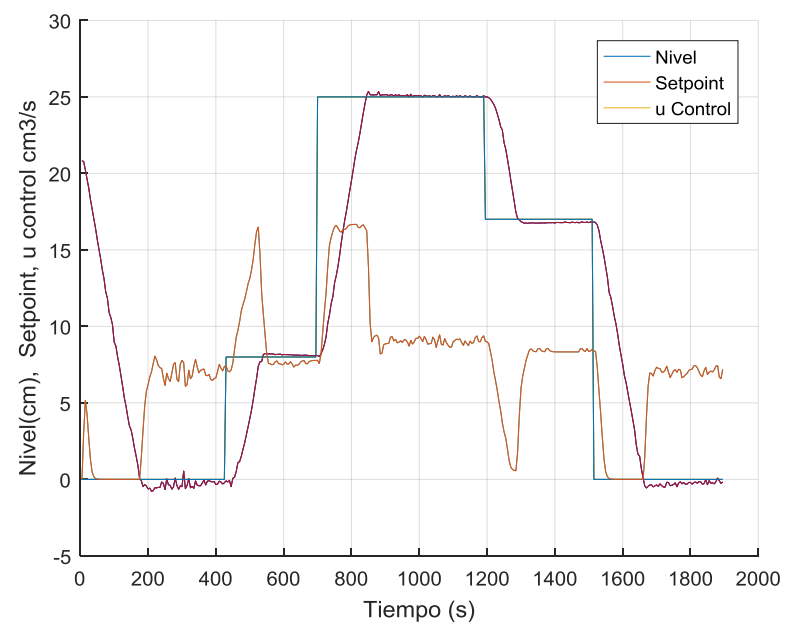

Fig. 4 Resultado prueba en plan real del controlador adaptativo operando el lazo simple sin perturbaciones.

El algoritmo lleva al nivel por lo diferentes valores de operación sesteados, no se observa sobre nivel porcentual, y el tiempo de estabilización es pequeño (2-3 minutos), comparado con el tiempo de respuesta de lazo abierto que esta entre $20 \mathrm{y}$ 30 minutos, los datos de nivel de salida y de control caudal (caudal/10) se consolidan en la figura 4.
Así mismo a la prueba de lazo simple se le aplica diferentes disturbios, ya sea en la entrada o salida, a través de la válvula para este fin (figura 1). Los datos de esta prueba se consolidan en la figura 5. En la que se nota que en los puntos donde se aplica las perturbaciones (100s, 500s y 600s), la planta pierde nivel y hasta recuperar el punto de operación el controlador tarda un tiempo significativo.

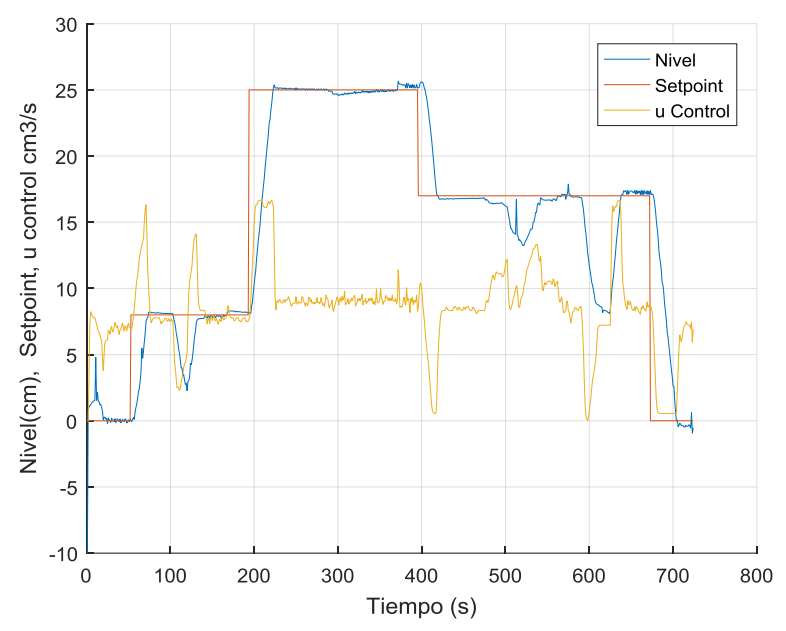

Fig. 5 Resultado prueba en plan real del controlador adaptativo operando el lazo simple con perturbaciones.

Luego de estas pruebas se habilita el lazo de control interno para que el sistema opere en doble lazo de control adaptativo. Se hace pasar por los mismos puntos de operación del caso anterior $(0 \mathrm{~cm}-8 \mathrm{~cm}-25 \mathrm{~cm}-17 \mathrm{~cm})$, al sistema, sin aplicarle perturbaciones, la respuesta del esta experimentación, son similares a los de la pruebas de lazo simple figura 4. Con la diferencia que la señal de control del lazo externo es el setpoint del lazo interno, este efecto se puede apreciar en las figuras $6 \mathrm{y}$ 7. En donde el controlador interno hace que el sistema sigue a la señal de control externa $(u)$.

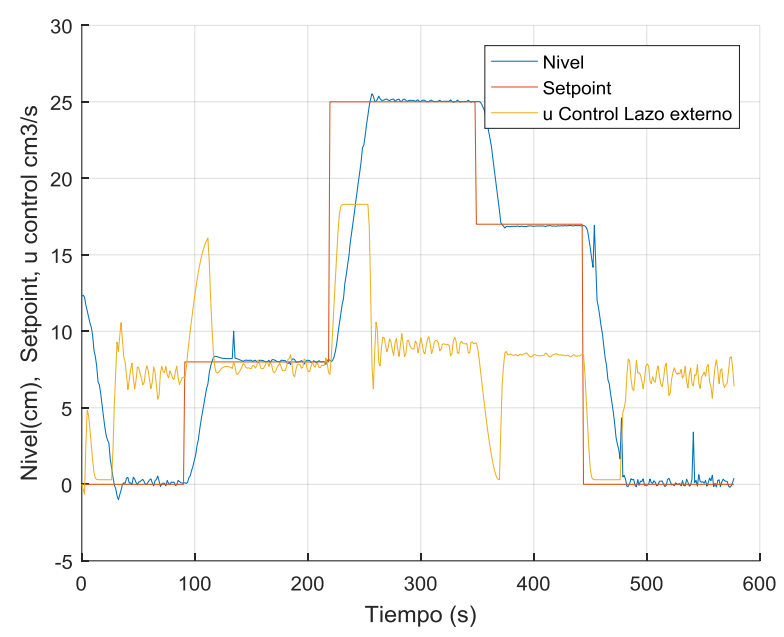

Fig. 6 Repuesta del controlador adaptativo operando el lazo cascada sin perturbaciones lazo externo.

$17^{\text {th }}$ LACCEI International Multi-Conference for Engineering, Education, and Technology: "Industry, Innovation, And Infrastructure for Sustainable Cities and Communities”, 24-26 July 2019, Jamaica. 


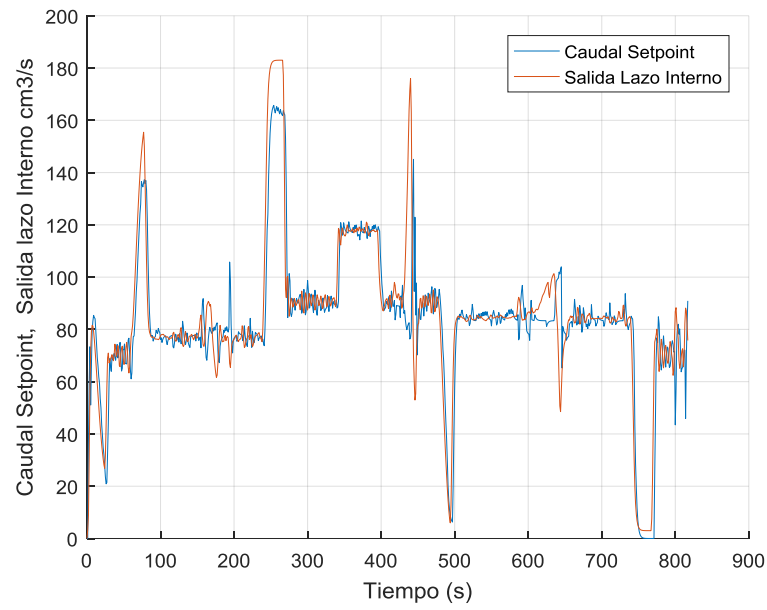

Fig. 6 Repuesta del controlador adaptativo operando el lazo cascada sin perturbaciones lazo interno.

En la prueba del control adaptativo de doble lazo se aplican perturbaciones, las consignas que se colocaron son similares a los de las pruebas precedentes, las respuestas del lazo externo e interno se presentan en las figuras 7 y 8 en las que se nota que el controlador cascada adaptativo logra corregir adecuadamente las perturbaciones y son imperceptibles en la salida de la planta(nivel), así mismo, el controlador interno sigue fielmente a la referencia u del controlador interno (figura 8 ), en los puntos en donde se aplica las perturbaciones se nota que el controlador interno cambia la u de control lo que hace que el control interno realice correcciones para mantener la salida en el nivel deseado, y finalmente en la figura 8 también se presenta la señal que se le envía al actuador, en la que se nota que en los puntos donde existen las perturbaciones el actuador debe aumentar la señal de control para compensar las pérdidas producto de las perturbaciones generadas en las pruebas.

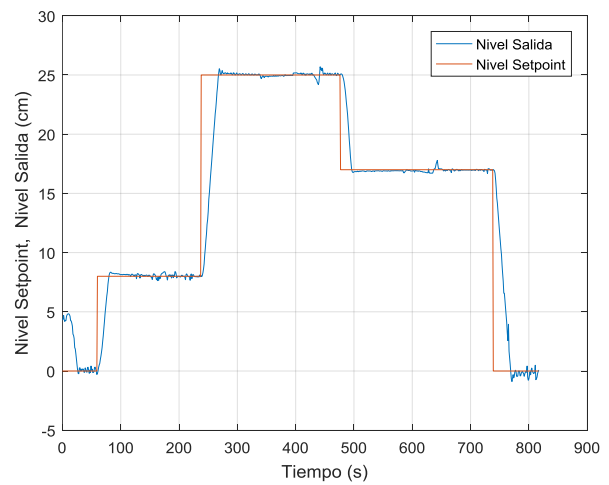

Fig. 7 Repuesta del controlador adaptativo operando el lazo cascada con perturbaciones lazo externo.

Al realizar la comparación de las respuestas del controlador adaptativo de lazo simple figura 5 con la respuesta de controlador adaptativo de lazo cascada figura 7, se nota que en el lazo simple, los efectos de las perturbaciones afectan significativamente a la salida del sistema, las perturbaciones hacen que el nivel de la planta baje y el control lo recupera, pero en un tiempo determinado, no así en el controlador adaptativo cascada de doble lazo, en donde las perturbaciones no afectan la salida, son insignificantes sus variaciones, prácticamente la planta mantiene el nivel deseado.

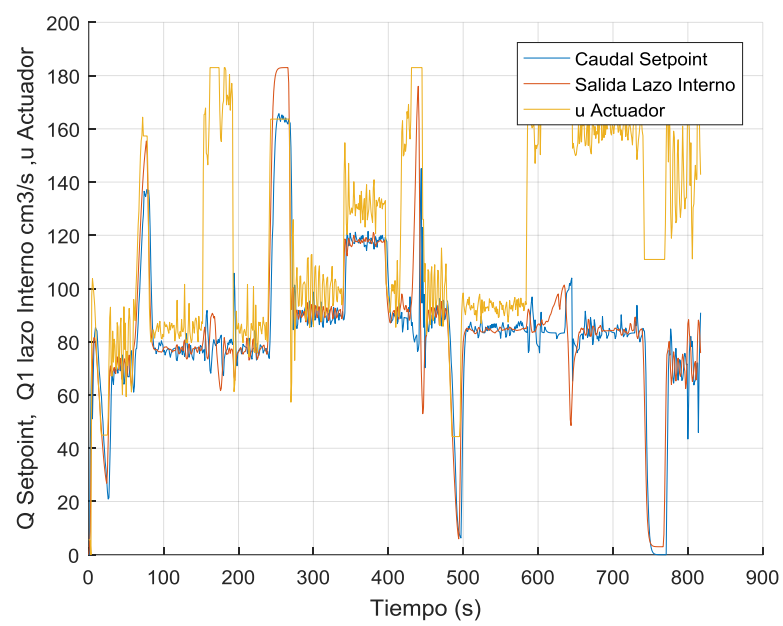

Fig. 8 Repuesta del controlador adaptativo operando el lazo cascada con perturbaciones lazo interno.

\section{CONCLUSIONES}

El modelo matemático tanto del tanque como del actuador, para realizar el control de nivel y caudal (ecuaciones 8, 9, 10,11), respectivamente, resultaron eficientes, permitieron calibrar las constantes de los controladores PID y PI del lazo externo e interno. Al comprobar el sistema con el algoritmo de control cascada adaptativo en la planta real si perturbaciones, el controlador logra llevarlo por diferentes puntos de operación, no se provocan sobre niveles porcentuales, y el tiempo de estabilización es muy bueno, alrededor de 2 o 3 minutos, al compararlo con la prueba de lazo abierto, que corresponde aproximadamente 30 minutos para un nivel de $38 \mathrm{~cm}$ del tanque de control, lo que asegura la buena calibración de los controladores adaptativos desarrollado en esta investigación.

Al realizar perturbaciones al equipo con lazo de control cascada, se pudo comprobar lo expuestos por la comunidad científica, que las perturbaciones en la entrada del proceso no se manifiestan en la salida, en la pruebas (figura 7) el nivel de la planta se mantienen a pesar de las perturbaciones efectuadas, la corrección de estas se debe a la activación del control de lazo interno (figura 8.) para compensar las pérdidas producto de las perturbaciones inyectadas en el equipo didáctico. Pruebas que en un control en lazo simple si afectaría significativamente a la variable se salida, como se pudo verificar en el equipo (figura 5) el nivel cae ante los disturbios que se provocaron.

\section{REFERENCIAS}

[1] L. Po, L. Ruiyu, S. Tianying, Z. Jingrui, and F. Zheng, "Composite adaptive model predictive control for DC-DC boost converters," IET Power Electron., vol. 11, no. 10, pp. 1706-1717, 2018.

[2] A. A. Goswami and B. T. Bezboruah, "Design and Implementation of an Embedded System for Monitoring and," J. Telecommun. Inf. Technol., pp. 23-32, 2012.

$17^{\text {th }}$ LACCEI International Multi-Conference for Engineering, Education, and Technology: "Industry, Innovation, And Infrastructure for Sustainable Cities and Communities", 24-26 July 2019, Jamaica. 
[3] V. Satyananda, K. V. Narayanaswamy, and K. Karibasappa, "An embedded system based solution for exudate extraction," Proceeding 2017 Int. Conf. Robot. Autom. Sci. ICORAS 2017, vol. 2018-March, pp. 1-5, 2018.

[4] J. Alvarez, L. Cordova, N. Vega, P. Parra, and J. Andramuno, "Cascade Control Algorithm developed with Embedded Systems," 2018 IEEE Int. Conf. Autom. Congr. Chil. Assoc. Autom. Control, pp. $1-6,2019$.

[5] A. J. Modak, S. B. Deshpande, and P. V. Dhawas, "New Hybrid Control Approach for Cascade Temperature Control in Catalyst Regeneration Process," no. 1, pp. 1-6, 2006.

[6] R. Hyl and R. Wagnerova, "Design and implementation of cascade control structure for superheated steam temperature control," 2016 17th Int. Carpathian Control Conf., pp. 253-258, 2016.

[7] J. M. Maciejowski, I. French, I. Fletcher, and T. Urquhart, "Cascade control of a process plant using predictive and multivariable control,", [1991] Proc. 30th IEEE Conf. Decis. Control, pp. 583-584 vol.1, 1991.

[8] L. Wei and F. Fang, "A novel IMC cascade control structure of superheated steam temperature system for super-critical coal-fired boilers," Proc. 2011 Chinese Control Decis. Conf. CCDC 2011, pp. 641-645, 2011.

[9] L. V. Bellinaso, H. H. Figueira, M. F. Basquera, R. P. Vieira, H. A. Grundling, and L. Michels, "Cascade Control with Adaptive Voltage Controller applied to Photovoltaic Boost Converters," IEEE Trans. Ind. Appl., vol. 9994, no. c, pp. 1-1, 2018.

[10] S. Chonsatidjamroen, K. N. Areerak, and K. L. Areerak, "The optimal cascade PI controller design of buck converters," 2012 9th Int. Conf. Electr. Eng. Comput. Telecommun. Inf. Technol. ECTI-CON 2012, pp. 1-4, 2012.

[11] R. M. E. F. Álvarez D. Juan Gonzalo Moreno S. Jorge Enrique, "Diseño e implementación de un sistema de control cascada en la planta de intercambio térmico - PIT000," Informado Técnico 81(1) Enero - Junio 2017, vol. 81, no. 1, pp. 32-43, 2017.

[12] J. Machuca, A \& Yépez, "Diseño, Construcción Y Experimentación De Un Viscosímetro Saybolt Y Desarrollo De Un Sistema De Control Temperatura En Ingeniero Mecánico Escuela Superior Politécnica De Chimborazo Facultad De Mecánica Escuela De Ingeniería Mecánica,” pp. 32-34, 2009.

[13] C. Lanping, "Adaptive stabilization for cascade nonlinear systems using the backstepping and control lyapunov function techniques," Fifth World Congr. Intell. Control Autom. (IEEE Cat. No.04EX788), vol. 1, no. 3, pp. 824-827, 2004.

[14] G. V. Raffo, J. E. Normey-Rico, F. R. Rubio, and C. R. Kelber, "Control Predictivo en Cascada de un Vehículo Autónomo," Rev. Iberoam. Automática e Informática Ind. RIAI, vol. 6, no. 1, pp. 6374, 2009 .

[15] L. Po, L. Ruiyu, S. Tianying, and Z. Jianfeng, "An improved adaptive cascade control for DC-DC boost converters," in Proceedings IECON 2017 - 43rd Annual Conference of the IEEE Industrial Electronics Society, 2017.

[16] S. C. C. Navarrete, "Control en cascada de la temperatura de un reactor continuo de tanque agitado encamisado Resumen," 2018.

[17] G. Ortiz-Torres, C. D. García-Beltran, J. Reyes-Reyes, A. VidalRosas, and C. M. Astorga-Zaragoza, "Control Tolerante a Fallas Pasivo de un Octorotor tipo X8 utilizando Controladores Backstepping en Cascada," XVI Conv. Ing. Eléctrica (CIE), St. Clara, Cuba, no. May, pp. 1-6, 2015.

[18] E. Hernández-Martínez, R. Urrea, and J. Álvarez-Ramírez, "Mejora En El Control En Cascada Para Reactores Tubulares Improved
Cascade Control Scheme for Tubular Reactors," Rev. Mex. Ing. Química, vol. 7, no. 3, pp. 309-318, 2008.

[19] W. Orozco-Tupacyupanqui, M. Nakano-Miyatake, and H. PérezMeana, "Filtro híbrido adaptativo en cascada para arreglos lineales uniformes de antenas," Inf. Tecnol., vol. 25, no. 4, pp. 103-122, 2014.

[20] V. M. Hung, I. Stamatescu, C. Dragana, and N. Paraschiv, "Comparison of model reference adaptive control and cascade PID control for ASTank2," in Proceedings of the 2017 IEEE 9th International Conference on Intelligent Data Acquisition and Advanced Computing Systems: Technology and Applications, IDAACS 2017, 2017.

[21] J. D. Grajales, D. F. López, J. E. Muñoz, and R. Maldonado, "CONTROL EN CASCADA POR J osé D avid G rajales *," Rev. EIA, pp. 181-193, 2007.

[22] F. Todeschini, M. Corno, G. Panzani, S. Fiorenti, and S. M. Savaresi, "Adaptive cascade control of a brake-by-wire actuator for sport motorcycles," IEEE/ASME Trans. Mechatronics, vol. 20, no. 3, pp. 1310-1319, 2015.

[23] L. Cordova, V. Navarrete, P. Parra, J. Andramuno, and N. Vega, "Adaptive Control Implemented with Embedded Systems," 2018 IEEE Int. Conf. Autom. Congr. Chil. Assoc. Autom. Control, pp. 1-6, 2019.

[24] N. Vega, P. Parra, and D. Martillo, "Didactic equipment developed with embedded systems for the learning of engineering," 2017 Chil. Conf. Electr. Electron. Eng. Inf. Commun. Technol. CHILECON 2017 - Proc., vol. 2017-January, pp. 1-6, 2017.

[25] S. A. J. Mary and J. A. Linsely, "Modeling and Control of Level Control Process- A Comparative Study," pp. 1-4.

[26] B. Bu, C. Li, and M. Chen, "Based on PLC fuzzy control algorithm in the application of level control," Proc. - 2016 IEEE Int. Symp. Comput. Consum. Control. IS3C 2016, pp. 698-701, 2016.

[27] M. S. Anand and B. Tyagi, "Design and Implementation of Fuzzy Controller on FPGA," Int. J. Intell. Syst. Appl., vol. 4, no. 10, pp. 3542, 2012.

[28] P. Pipitsunthonsan, P. Jawayon, and T. Khaorapapong, "The modeling of screw pump dispenser using DC motor," 2016 3rd Int. Conf. Electron. Des. ICED 2016, pp. 236-239, 2017.

[29] P. Panchal, A. Patel, and J. Barve, "PI control of level control system using PLC and LabVIEW based SCADA," 2015 Int. Conf. Ind. Instrum. Control. ICIC 2015, no. Icic, pp. 1196-1201, 2015.

[30] P. Keadtipod and D. Banjerdpongchai, "Supervisory Robust Model Predictive Control for Linear Systems Subject to Parametric Uncertainty and Application to Level Control Process," 2018 15th Int. Conf. Electr. Eng. Comput. Telecommun. Inf. Technol., pp. 680 683, 2019.

[31] Q. Zhu, G. Li, and R. Zhou, "Integrated model of water pump and electric motor based on BP neural network," Proc. 2015 10th IEEE Conf. Ind. Electron. Appl. ICIEA 2015, pp. 1449-1452, 2015.

[32] W. U. Qinghui, "Research on Modeling of Water Supply System based on VVVF Speed Regulation," Proc. 31st Chinese Control Conf., pp. 1817-1819, 1817.

[33] M. Akbaba and M. C. Akbaba, "Dynamic performance of a photovoltaic-boost converter powered DC motor-pump system," IEMDC 2001 - IEEE Int. Electr. Mach. Drives Conf., pp. 356-361, 2001.

[34] W. Tan and H. Zhang, "Simple gain-scheduled water level control for nuclear steam generator," Proc. 29th Chinese Control Conf. CCC'10, vol. 1, no. 1, pp. 4969-4974, 2010.

$17^{\text {th }}$ LACCEI International Multi-Conference for Engineering, Education, and Technology: "Industry, Innovation, And Infrastructure for Sustainable Cities and Communities", 24-26 July 2019, Jamaica. 